\title{
Prevalence of and Risk Factors for Diabetic Retinopathy and Diabetic Macular Edema in Patients with Early- and Late-Onset Diabetes Mellitus
}

\author{
Yu Wang ${ }^{a}$ Zhong Lin ${ }^{b}$ Gang Zhai ${ }^{a}$ Xiao Xia Ding ${ }^{a}$ Liang Wen ${ }^{a}$ Dong Li ${ }^{a}$ \\ Bo Zou $^{a}$ Ke Mi Feng ${ }^{b}$ Yuan Bo Liang ${ }^{b}$ Cong Xie $^{a}$

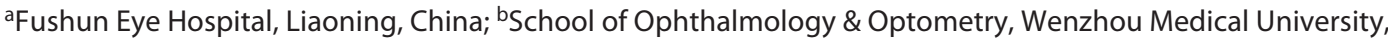 \\ Eye Hospital of Wenzhou Medical University, Wenzhou, China
}

\section{Keywords \\ Diabetes - Age of onset - Diabetic retinopathy - Diabetic macular edema}

\begin{abstract}
Purpose: The aim of this study was to compare the prevalence of diabetic retinopathy (DR) and diabetic macular edema (DME), as well as their risk factors in patients with earlyonset diabetes (EOD, $\leq 40$ years) and late-onset diabetes (LOD, >40 years). Methods: Patients were recruited from a community-based study, Fushun Diabetic Retinopathy Cohort Study (FS-DIRECT), conducted between July 2012 and May 2013 in China. The presence and severity of DR and DME were determined by a modified Early Treatment Diabetic Retinopathy Study (ETDRS) retinopathy scale of six-field fundus photographs. Results: A total of 1,932 patients (796 male, $41.2 \%$ ) with gradable fundus photography were included. The prevalence of any DR and DME was $67.0 \%(95 \%$ confidence interval [Cl]: 60.3-73.7\%) and 39.3\% $(95 \% \mathrm{Cl}$ : $32.1-46.5 \%)$ in the EOD patients, respectively, which were both significantly higher than that in the LOD patients (DR: 41.9\%, 39.6-44.2\%, $p<0.001$; DME: 14.4\%, 12.7-16.1\%, $p<$ $0.001)$. Insulin use was associated with both the presence of DR and DME in both EOD and LOD patients. Besides insulin
\end{abstract}

Karger@karger.com www.karger.com/ore

Karger!"

BOPEN ACCESS
(C) 2020 The Author(s)

Published by S. Karger AG, Basel

This is an Open Access article licensed under the Creative Commons Attribution-NonCommercial-4.0 International License (CC BY-NC) (http://www.karger.com/Services/OpenAccessLicense), applicable to the online version of the article only. Usage and distribution for commercial purposes requires written permission. use, a high level of income (odds ratio [OR], $95 \% \mathrm{Cl}$ : 0.05, 0.01-0.51) was negatively associated with DR, and higher high-density lipoprotein (OR, 95\% Cl: 4.14, 1.44-11.91) was associated with DME among EOD patients. Conclusion: In this sample of patients with type 2 diabetes, both prevalence of DR and DME were apparently higher in patients who developed diabetes $\leq 40$ years of age than those who developed it later.

(c) 2020 The Author(s) Published by S. Karger AG, Basel

\section{Introduction}

Diabetes has become a global health and economic problem, especially in low- and middle-income countries [1]. As the largest developing and most populous country, it was estimated that more than 92 million and 148 million Chinese adults were having diabetes and prediabetes [2]. Evidence from the Indian population shows that the highest prevalence of new-onset diabetes tends toward a younger age $[3,4]$. Patients who develop diabetes at an early age have a longer exposure to hyperglycemia which

Yu Wang and Zhong Lin contributed equally to this work.
Correspondence to:

Gang Zhai, fsyanbing@163.com 
leads to higher prevalence and more severe microvascular complications, such as diabetic retinopathy (DR) [5-11], and even mortality [12]. Hence, a better understanding of the prevalence of DR and its associated risk factors among patients with different ages of diabetes onset is of paramount importance.

Previous population-based study from urban Indian areas showed that the prevalence of DR was almost twice in patients who developed diabetes $\leq 40$ years of age than those who developed it later (33.3\% vs. $15.6 \%$ ) [9]. However, more recent data from a rural Indian population showed only a slightly higher prevalence of $\mathrm{DR}$ in patients with known diabetes $\leq 40$ years of age ( $11.5 \%$ vs. $10.0 \%)$ [11]. However, similar data from Chinese population are rare. Recently, we reported a very high prevalence of DR (44.3\%) among type 2 diabetes patients in a northeastern Chinese population [13]. The comparison between the prevalence of DR and its complications may prove to be useful and valuable for the future. In this study, the prevalence of DR and DME as well as their risk factors among patients with a known onset of diabetes before and after 40 years are reported.

\section{Methods}

Patients from the Fushun Diabetic Retinopathy Cohort Study (FS-DIRECT), conducted between July 2012 and May 2013 in Fushun city, Liaoning Province, China, were enrolled. The study design and methodology of the FS-DIRECT were reported elsewhere [13]. Briefly, residents aged 30 years and above in all of the communities of Jiangjun Street, Fushun City, with type 2 diabetes were recruited. Detailed examinations, blood sample collection, and questionnaires were performed for all patients. The age of the known onset of diabetes was calculated by subtracting the known duration of diabetes from the age at presentation.

The inclusion criteria for this study were available data of the level of DR and available data of age of the diabetes onset. Ethics Committee approval was obtained from the Fushun Eye Hospital. Written informed consent was obtained from all subjects.

\section{Definition of Diabetes}

Diabetes was diagnosed according to the criteria of American Diabetes Association [14]: fasting plasma glucose (FPG) $\geq 7.0$ $\mathrm{mmol} / \mathrm{L}$ or 2 -h oral glucose tolerance test $\geq 11.1 \mathrm{mmol} / \mathrm{L}$ or selfreported diabetes medication use. DM was considered to be type 1 if the participant was aged less than 30 years when diagnosed with diabetes and was receiving insulin therapy. Otherwise, DM was considered to be type 2 .

\section{Evaluation of DR and DME}

Graders were trained and certified at the Retinal Vascular Imaging Centre, University of Melbourne, before the assessment of photographs. Fundus photographs of 6 fields were graded in a masked manner by two well-trained graders according to the mod- ified Airlie House Classification system [15]. The grading procedure was consistent with previous population-based or community-based studies in China [16-18]. If there was a disagreement in the retinopathy or macula edema level assigned, the eye will be sent to a third grader for an edited grade. The level of retinopathy and ME was based on the grading of the worse eye. If an eye was unable to grade, the scores for the other eye were used. The level of retinopathy was graded according to the following criteria: (1) no DR (levels 10-20), (2) nonproliferative DR (NDR) (mild [levels 3137], moderate [levels 43-47], or severe [levels 53]), or (3) proliferative DR (levels 60-85). DME was defined as the presence of retinal thickening within 1 disk diameter from the foveal center or the presence of focal photocoagulation scars in the macular area. Clinically significant macular edema (CSME) was considered present when one of the following situation was present: (1) retinal thickening within $500 \mu \mathrm{m}$ from the center of the macula or focal photocoagulation scars were present; (2) hard exudates within 500 $\mu \mathrm{m}$ from the center of the macula with adjacent retinal thickening; and (3) retinal thickening of more than one optic disc area within one optic disc diameter from the center of the macula. The grading process was completed within 1 month. The unweighted $\kappa$ statistics, calculated for intergrader and intragrader agreement on a randomly selected subset of 100 participants stratified by the retinopathy severity level, were 0.98 and 0.91 , respectively, indicating excellent agreement.

\section{Statistical Analysis}

The normally distributed parameters were presented as the mean \pm standard deviation and compared by independent $t$-tests. $\chi^{2}$ tests or Fisher's exact tests (if necessary) were performed for the comparison of the discrete categorized data. Risk factors, including age, gender, education level, income level, duration of diabetes, treatment age of diabetes, current smoker, current drinker, use of insulin, FPG, glycosylated hemoglobin A1c (HbA1c), body mass index, waist-hip ratio (WHR), systolic blood pressure (SBP), diastolic blood pressure, serum creatinine, blood urea nitrogen, blood uric acid, total cholesterol, total triglycerides, low-density lipoprotein (LDL), high-density lipoprotein (HDL), and the presence of proteinuria, were primarily assessed in the univariate regression for the association with DR and DME, respectively. Age, gender, as well as risk factors with a statistical $p$ value less than 0.1 were further analyzed in multivariate logistic regressions. Statistical analyses were performed using Statistical Analysis System for Windows version 9.1.3 (SAS Inc., Cary, NC, USA). A $p$ value less than 0.05 was considered to be statistically significant.

\section{Results}

A total of 1,932 patients (796 male, $41.2 \%$ ) with gradable fundus photography and known age of the diabetes onset were included in this study. Among them, 188 patients $(9.7 \%)$ had diabetes at $\leq 40$ years of age (early-onset diabetes, EOD), with a mean age of $36.7 \pm 3.0$ (range $21-$ 40) years. The rest of the patients had diabetes $>40$ years of age (late-onset diabetes, LOD), with a mean age of 56.0 \pm 8.4 (range $41-82$ ) years. 
Table 1. Comparison of characteristics between EOD and LOD

\begin{tabular}{|c|c|c|c|}
\hline & $\operatorname{EOD}(n=188)$ & $\operatorname{LOD}(n=1,744)$ & $p$ value \\
\hline Age, years & $50.0 \pm 7.3$ & $63.0 \pm 8.0$ & $<0.001$ \\
\hline Male, $n(\%)$ & $93(49.5)$ & $703(40.3)$ & 0.02 \\
\hline Age of diabetic onset, years & $36.7 \pm 3.0$ & $56.0 \pm 8.4$ & $<0.001$ \\
\hline Duration of diabetes, years & $13.3 \pm 7.3$ & $7.0 \pm 5.4$ & $<0.001$ \\
\hline Interval between treatment and diagnosis, years & $2.1 \pm 4.7$ & $1.1 \pm 2.9$ & 0.008 \\
\hline \multicolumn{4}{|l|}{ Monthly income (CNY), $n$ (\%) } \\
\hline $\operatorname{Low}(<3,000)$ & $104(57.5)$ & $605(35.5)$ & \multirow[t]{3}{*}{$<0.001$} \\
\hline Moderate $(3,000-5,000)$ & $66(36.5)$ & $1,022(60.0)$ & \\
\hline High $(\geq 5,000)$ & $11(6.1)$ & $76(4.5)$ & \\
\hline \multicolumn{4}{|l|}{ Education, $n(\%)$} \\
\hline Primary or lower & $7(3.9)$ & $292(17.1)$ & \multirow[t]{3}{*}{$<0.001$} \\
\hline Middle/high school & $144(79.1)$ & $1,181(69.1)$ & \\
\hline College and above & $31(17.0)$ & $237(13.9)$ & \\
\hline Current cigarette smoker, $n(\%)$ & 65 (35.9) & $350(20.5)$ & $<0.001$ \\
\hline Current alcohol drinker, $n(\%)$ & $66(36.5)$ & $350(20.5)$ & $<0.001$ \\
\hline \multicolumn{4}{|l|}{ Diabetes treatment, $n(\%)$} \\
\hline Insulin user, $n(\%)$ & $91(50.0)$ & $371(21.9)$ & $<0.001$ \\
\hline $\mathrm{FPG}, \mathrm{mmol} / \mathrm{L}$ & $11.7 \pm 4.6$ & $9.1 \pm 3.2$ & $<0.001$ \\
\hline $\mathrm{HbA} 1 \mathrm{C}, \%$ & $8.7 \pm 2.3$ & $7.7 \pm 2.0$ & $<0.001$ \\
\hline $\mathrm{BMI}, \mathrm{kg} / \mathrm{m}^{2}$ & $26.30 \pm 3.20$ & $26.41 \pm 3.44$ & 0.65 \\
\hline WHR & $0.95 \pm 0.06$ & $0.97 \pm 0.07$ & 0.01 \\
\hline $\mathrm{SBP}, \mathrm{mm} \mathrm{Hg}$ & $143.4 \pm 22.7$ & $148.1 \pm 23.2$ & 0.01 \\
\hline $\mathrm{DBP}, \mathrm{mm} \mathrm{Hg}$ & $81.0 \pm 12.5$ & $76.9 \pm 11.2$ & $<0.001$ \\
\hline Serum creatinine, $\mu \mathrm{mol} / \mathrm{L}$ & $84.14 \pm 20.34$ & $84.85 \pm 19.50$ & 0.64 \\
\hline Blood urea nitrogen, $\mathrm{mmol} / \mathrm{L}$ & $6.07 \pm 1.92$ & $6.27 \pm 3.30$ & 0.22 \\
\hline Blood uric acid, $\mu \mathrm{mol} / \mathrm{L}$ & $303.33 \pm 80.32$ & $310.23 \pm 88.30$ & 0.31 \\
\hline Total cholesterol, $\mathrm{mmol} / \mathrm{L}$ & $5.54 \pm 1.21$ & $5.51 \pm 1.26$ & 0.81 \\
\hline Total triglycerides, $\mathrm{mmol} / \mathrm{L}$ & $2.69 \pm 2.41$ & $2.15 \pm 1.55$ & 0.004 \\
\hline $\mathrm{LDL}, \mathrm{mmol} / \mathrm{L}$ & $3.36 \pm 1.01$ & $3.22 \pm 0.92$ & 0.05 \\
\hline $\mathrm{HDL}, \mathrm{mmol} / \mathrm{L}$ & $1.47 \pm 0.38$ & $1.48 \pm 0.37$ & 0.54 \\
\hline Presence of proteinuria, $n(\%)$ & $71(37.8)$ & $571(32.8)$ & 0.17 \\
\hline
\end{tabular}

EOD, early-onset diabetes; LOD, late-onset diabetes; CNY, China Yuan; FPG, fasting plasma glucose; HbA1c, glycosylated hemoglobin A1c; BMI, body mass index; WHR, waist-hip ratio; SBP, systolic blood pressure; DBP, diastolic blood pressure; LDL, low-density lipoprotein; HDL, high-density lipoprotein.

The characteristics and corresponding comparisons between EOD and LOD patients were presented in Table 1 . The EOD patients were younger $(p<0.001)$, comprised of more males $(p=0.02)$, longer duration of DM $(p<0.001)$, longer interval between diagnosis and treatment of DM ( $p=0.008)$, more current cigarette smokers and alcohol drinkers (both $p<0.001$ ), more insulin users $(p<0.001)$, higher FPG and HbA1C (both $p<0.001$ ), smaller waist-hip ratio $(p=0.01)$, lower SBP $(p=0.01)$, and higher diastolic blood pressure $(p<0.001)$, as compared to LOD patients. It was also found that the EOD patients had higher proportion of high income levels and high education levels (both $p<0.001$ ).

The prevalence of any DR and DEM was 67.0\% (95\% confidence interval [CI]: 60.3-73.7\%) and 39.3\% (95\%
CI: 32.1-46.5\%) in the EOD patients, both significantly higher than that in the LOD patients (DR: 41.9\%, 39.6$44.2 \%, p<0.001$; DME: $14.4 \%, 12.7-16.1 \%, p<0.001)$. When further dividing patients into subgroups according to the severity of DR and DME, it was found that EOD patients tended to have both more severe types of DR and DME $(p<0.001)$ (Table 2). Table 3 shows the EOD patients are consistently more likely to have more severe DR-related pathologic features (all $p<0.05$ ).

In a multivariate logistic regression for any DR among EOD patients, adjusting for age, gender, duration of DM, income level, use of insulin, FPG, HbAlc, body mass index, SBP, blood urea nitrogen, HDL, and the presence of proteinuria, selected from univariate regressions, two risk factors remained significant, i.e., high income level (odds 
Table 2. Prevalence ( $n, \%)$ of DR and DME between EOD and LOD

\begin{tabular}{llll}
\hline & $\operatorname{EOD}(n=188)$ & $\operatorname{LOD}(n=1,744)$ & $p$ value \\
\hline DR & & & \\
$\quad$ Any DR & $126(67.0)$ & $730(41.9)$ & $<0.001$ \\
Mild NPDR & $53(28.2)$ & $528(30.3)$ & $<0.001$ \\
$\quad$ Moderate NPDR & $23(12.2)$ & $85(4.9)$ & \\
$\quad$ Severe NPDR & $20(10.6)$ & $63(3.6)$ & \\
$\quad$ PDR & $30(16.0)$ & $54(3.1)$ & \\
DME* & $33(18.5)$ & $119(6.9)$ & $<0.001$ \\
$\quad$ Not CSME & $37(20.8)$ & $129(7.5)$ & \\
$\quad$ CSME & &
\end{tabular}

DR, diabetic retinopathy; DME, diabetic macular edema; EOD, early-onset diabetes mellitus; LOD, late-onset diabetes mellitus; NPDR, nonproliferative diabetic retinopathy; PDR, proliferative diabetic retinopathy; CSME, clinically significant macular edema. DME*: 178 and 1721 patients with gradable fundus photography in EOD and LOD groups, respectively.

ratio [OR], 95\% CI: $0.05,0.01-0.51)$ and use of insulin (OR, 95\% CI: 2.56, 1.12-5.84). For LOD patients, adjusting for age, gender, duration of DM, education level, current cigarette smoker, current alcohol drinker, use of insulin, FPG, HbA1c, SBP, total cholesterol, HDL, and the presence of proteinuria, significant risk factors remaining were age, duration of DM, use of insulin, FPG, HbA1c, SBP, and the presence of proteinuria (Table 4).

Regarding the DME among EOD patients, use of insulin (OR, 95\% CI: 2.61, 1.18-5.77) and HDL (OR, 95\% CI: $4.14,1.44-11.91)$ were significantly associated with the presence of DME, after adjusting for age, gender, duration of DM, income level, current drinker, use of insulin, FPG, HbA1c, SBP, blood urea nitrogen, total cholesterol, HDL, and the presence of proteinuria. For LOD patients, age, duration of DM, current alcohol drinker, use of insulin, SBP, total cholesterol, and the presence of proteinuria remained significant, after adjusting for age, gender, duration of DM, current cigarette smoker, current alcohol drink, use of insulin, FPG, HbA1c, SBP, serum creatinine, total cholesterol, total triglycerides, LDL, and the presence of proteinuria (Table 4).

\section{Discussion}

In this community-based study, nearly $10 \%$ of the patients developed diabetes at the age of 40 or younger (mean 36.7 years) among the northeastern Chinese population with type 2 diabetes. As expected, the prevalence of
Table 3. Pathological features, $n$ (\%) of DR between EOD and LOD

\begin{tabular}{|c|c|c|c|}
\hline & EOD & LOD & $p$ value \\
\hline \multicolumn{4}{|c|}{ MA and spot hemorrhage } \\
\hline None & $32(17.4)$ & $690(39.7)$ & $<0.001$ \\
\hline Mild & $115(62.5)$ & $917(52.7)$ & \\
\hline Severe & $37(20.1)$ & $133(7.6)$ & \\
\hline \multicolumn{4}{|c|}{ Hard exudation } \\
\hline None & $111(60.3)$ & 1,377 (79.3) & $<0.001$ \\
\hline Presence & $73(39.7)$ & $360(20.7)$ & \\
\hline \multicolumn{4}{|c|}{ Cotton wool spot } \\
\hline None & $121(65.8)$ & $1,521(87.8)$ & $<0.001$ \\
\hline Presence & $63(34.2)$ & $212(12.2)$ & \\
\hline \multicolumn{4}{|l|}{ IRMA } \\
\hline None & $150(82.0)$ & $1,670(96.4)$ & $<0.001$ \\
\hline Mild & $16(8.7)$ & $41(2.4)$ & \\
\hline Severe & $17(9.3)$ & $22(1.3)$ & \\
\hline \multicolumn{4}{|l|}{ VB* } \\
\hline None & $176(96.2)$ & $1,710(98.7)$ & 0.02 \\
\hline Presence & $7(3.8)$ & $23(1.3)$ & \\
\hline
\end{tabular}

$\mathrm{DR}$, diabetic retinopathy; EOD, early-onset diabetes mellitus; LOD, late-onset diabetes mellitus; MA, microaneurysm; IRMA, intraretinal microvascular abnormality; $V B$, venous beading. * Calculated by Fisher's exact test.

both DR and DME in the EOM patients was apparently higher than that in LOM patients, with 1.5 times for the prevalence of DR, and strikingly almost 3 times for the prevalence of DME. These distinct differences were further verified by the subgroups of DR and DME, where moderate NPDR, severe NPDR, proliferative diabetic retinopathy, and CSME were consistently higher in EOD patients. Moreover, EOD patients were consistently more likely to have or have more severe DR-related pathologic features, including microaneurysm, hard exudation, cotton wool spot, intraretinal microvascular abnormality, and venous beading.

This finding was consistent with previous studies. In the 1980s, Hamman et al. [5] found that younger age at diagnosis of diabetes was an independent risk factor for DR due to longer exposure to an increased glycosylated hemoglobin level. Haffner et al. [6] also reported that in both Mexican Americans and Caucasians, earlier age at diagnosis of diabetes was significantly associated with DR. Wong et al. [7] confirmed that the onset age of type 2 diabetes was independently associated with increasing DR risk in patients with long (OR and 95\% CI: 0.88, 0.830.94 ) and short (OR and 95\% CI: 0.7, 0.6-0.9) duration of diabetes. In the recent 2010s, in an urban Indian population, Ramen et al. [9] reported that the prevalence of DR 
Table 4. Significant risk factors $(\mathrm{OR}, 95 \% \mathrm{Cl})$ for any $\mathrm{DR}$ and $\mathrm{DME}$ in multivariate logistic regression in EOD and LOD

\begin{tabular}{|c|c|c|c|c|}
\hline & \multicolumn{2}{|l|}{$\mathrm{DR}$} & \multicolumn{2}{|l|}{ DME } \\
\hline & EOD & LOD & EOD & LOD \\
\hline Age, years & - & $0.97(0.95,0.98)$ & - & $0.93(0.91,0.95)$ \\
\hline Duration of diabetes, years & - & $1.17(1.14,1.20)$ & - & $1.14(1.10,1.17)$ \\
\hline \multicolumn{5}{|l|}{ Monthly income (CNY) } \\
\hline $\operatorname{Low}(<3,000)$ & 1.00 & - & - & - \\
\hline Moderate $(3,000-5,000)$ & $0.82(0.36,1.88)$ & - & - & - \\
\hline High $(\geq 5,000)$ & $0.05(0.01,0.51)$ & - & - & - \\
\hline Current alcohol drinker versus not current alcohol drinker & - & - & - & $0.51(0.30,0.86)$ \\
\hline Use of insulin & $2.56(1.12,5.84)$ & $2.05(1.55,2.71)$ & $2.61(1.18,5.77)$ & $2.00(1.41,2.82)$ \\
\hline $\mathrm{FPG}, \mathrm{mmol} / \mathrm{L}$ & - & $1.07(1.02,1.12)$ & - & - \\
\hline $\mathrm{HbA} 1 \mathrm{C}, \%$ & - & $1.10(1.02,1.18)$ & - & - \\
\hline $\mathrm{SBP}, \mathrm{mm} \mathrm{Hg}$ & - & $1.02(1.01,1.03)$ & - & $1.01(1.00,1.02)$ \\
\hline Total cholesterol, $\mathrm{mmol} / \mathrm{L}$ & - & - & - & $1.01(1.00,1.02)$ \\
\hline $\mathrm{HDL}, \mathrm{mmol} / \mathrm{L}$ & - & - & $4.14(1.44,11.91)$ & - \\
\hline Presence of proteinuria & - & $1.47(1.15,1.87)$ & - & $1.54(1.10,2.16)$ \\
\hline
\end{tabular}

OR, odds ratio; $\mathrm{Cl}$, confidence interval; $\mathrm{DR}$, diabetic retinopathy; $\mathrm{DME}$, diabetic macular edema; $\mathrm{EOD}$, early-onset diabetes mellitus; $\mathrm{LOD}$, late-onset diabetes mellitus; CNY, China Yuan; FPG, fasting plasma glucose; HbA1c, glycosylated hemoglobin A1c; SBP, systolic blood pressure; HDL, high-density lipoprotein.

was twice $(33.3 \%$ vs. $15.6 \%)$ in patients who developed diabetes at 40 years or younger as compared to those who developed it after 40 years. In a hospital-based, cross-sectional study in Chinese patients with type 2 diabetes, Zou et al. [10] also illustrated this effect of age of the diabetes onset (OR and 95\% CI: 0.985, 0.977-0.992) and further concluded that the age of onset at 31-45 years was an independent risk factor for DR (OR and 95\% CI: 1.815, 1.139-2.892). However, recently, from a rural Indian population, Khan et al. [11] found only a slightly higher prevalence of DR in patients with diabetes developed 40 years or younger $(11.5 \%$ vs. $10.0 \%)$. The authors explained this close prevalence due to the change in lifestyle in rural younger populations, as well as increasing awareness about diabetes and high proportion (60\%) of regular medical care in rural populations in recent years [11].

In the present study, after univariate selections of the risk factors and multivariate logistic regression, we found the insulin use was the only common risk factors for DR and DME in different ages of onset groups. This was consistent with previous studies, such as those carried out in the Indian population, where Raman et al. [9] and Khan et al. [11] both found the insulin use to be a risk factor for DR in different age of onset groups $[9,11]$. In a metaanalysis which included 14 studies involving 202,905 individuals, Zhang et al. [19] reported that the insulin use was associated with increased risk of macular edema (rel-

DR and DME in Early- and Late-Onset Diabetes Mellitus ative risk, 95\% CI: 3.42, 2.42-4.83). Besides the insulin use, patients with a high income level were less likely to have DR in the EOD patients. In a community-based study, Li et al. [18] reported that a lower income level was associated with the presence of DR (OR, 95\% CI: 1.49, 1.10-2.03) in Chinese type 2 diabetes patients. The high income level bracket may afford protection against DR via access to more frequent and possibly more effective medical care.

In the present study, HDL was associated with the presence of DME in EOD patients, besides the insulin use. In a population-based study, Raman et al. [20] found that high LDL, high non-HDL, and high cholesterol ratio were associated with non-CSME, and high total serum cholesterol was significant for CSME. However, neither association was found for HDL [20]. In the Wisconsin Epidemiologic Study of Diabetic Retinopathy, Klein et al. [21] did not find an association between total serum cholesterol or HDL and the incidence of DR or DME in type 1 diabetes. In a case-control study with multi-ethnicities (Malay, Chinese, and Indian), Jew et al. [22] did not find the association between HDL and the presence of CSME in patients with type 2 diabetes either. The difference in this study and previous studies on the HDL and DME may be due to different types of diabetes, ethnicities, and most importantly, taking account of the onset of age of diagnosis into consideration. 
The mechanisms of the higher prevalence of DR and DME among young-onset diabetic patients remained unknown. Investigations suggested the possible mechanisms include more active expression of the vascular endothelial growth factor in younger patients [23], "metabolic memory," or irreversible vascular damage after a long-term history of uncontrolled hyperglycemia among insouciant young patients [24-26] and a higher level of psychological pressure among young- and middle-aged working people $[27,28]$.

The advantages of this study included relatively large sample size, standard fundus photography and grading protocols, comprehensive questionnaires, and biological specimens. However, three main limitations of this study were identified and must be acknowledged. First, the cross-sectional data cannot identify cause-effect relationships. Further evidence is warranted in this cohort study. Second, the cutoff for the early onset of diabetes at 40 years of age was arbitrarily chosen in order to maximize the sample size in this group, as well as suggested in previous studies $[9,11]$. Hence, great caution should be given when generalizing the conclusion to patients with young-onset diabetes. Third, antihypertension and antihyperlipidemia therapies were not available in this study, and thus, certain bias remained for some risk factors, e.g., blood pressure, total cholesterol, and HDL.

In conclusion, in this sample of northeastern Chinese patients with type 2 diabetes mellitus, patients who developed diabetes at an earlier age tended to have a higher prevalence of both DR and DME. Identifying risk factors would be beneficial for preventing vision loss among young people with diabetes.

\section{Acknowledgment}

The authors thank Dr. Nived Moonasar (Caribbean Eye Institute, Valsayn, Trinidad and Tobago) for his invaluable assistance in revising the manuscript.

\section{Statement of Ethics}

Ethics Committee approval was obtained from the Fushun Eye Hospital. Written informed consent was obtained from all subjects.

\section{Conflict of Interest Statement}

The authors declare that there are no conflicts of interest. This manuscript has not been published anywhere previously, and it is not simultaneously being considered for any other publication.

\section{Funding Sources}

The study was supported by Wenzhou Basic Medical and Health Technology Project (Y2020364), Zhejiang Provincial Natural Science Foundation of China (LQ18H120004), and Liaoning Provincial Natural Science Foundation of China (20170540328).

\section{Author Contributions}

G.Z., Y.B.L., and C.X. designed the study protocol and conducted the study as a supervisor. Y.W., X.X.D., L.W., and D.L. participated in the study design. X.X.D., L.W., D.L., and B.Z. collected the study data. Z.L. and K.M.F. conducted statistical analysis. Y.W. and Z.L. drafted the manuscript. Z.L., K.M.F., and L.W. revised the manuscript. All the authors read and approved the final manuscript.

\section{References}

1 Guariguata L, Whiting DR, Hambleton I, Beagley J, Linnenkamp U, Shaw JE. Global estimates of diabetes prevalence for 2013 and projections for 2035. Diabetes Res Clin Pract. 2014;103:137-49.

2 Yang W, Lu J, Weng J, Jia W, Ji L, Xiao J, et al. Metabolic disorders study group: prevalence of diabetes among men and women in china. N Eng J Med. 2010;362:1090-101.

3 Mohan V, Deepa M, Deepa R, Shanthirani CS, Farooq S, Ganesan A, et al. Secular trends in the prevalence of diabetes and impaired glucose tolerance in urban south india-the chennai urban rural epidemiology study (cures-17). Diabetologia. 2006;49:1175-8.

4 Singla R, Garg A, Singla S, Gupta Y. Temporal change in profile of association between diabetes, obesity, and age of onset in urban india: a brief report and review of literature. Indian J Endocrinol Metab. 2018;22:429-32.
5 Hamman RF, Mayer EJ, Moo-Young GA, Hildebrandt W, Marshall JA, Baxter J. Prevalence and risk factors of diabetic retinopathy in non-hispanic whites and hispanics with nid$\mathrm{dm}$. San luis valley diabetes study. Diabetes. 1989;38:1231-7.

6 Haffner SM, Mitchell BD, Moss SE, Stern MP, Hazuda HP, Patterson J, et al. Is there an ethnic difference in the effect of risk factors for diabetic retinopathy? Ann Epidemiol. 1993;3:2-8.

7 Wong J, Molyneaux L, Constantino M, Twigg SM, Yue DK. Timing is everything: age of onset influences long-term retinopathy risk in type 2 diabetes, independent of traditional risk factors. Diabetes care. 2008;31:1985-90.

8 Lee BK, Kim SW, Choi D, Cho EH. Comparison of age of onset and frequency of diabetic complications in the very elderly patients with type 2 diabetes. Endocrinol Metab. 2016;31: 416-23.
9 Raman R, Vaitheeswaran K, Vinita K, Sharma $\mathrm{T}$. Is prevalence of retinopathy related to the age of onset of diabetes? Sankara nethralaya diabetic retinopathy epidemiology and molecular genetic report no. 5. Ophthalmic Res. 2011;45:36-41.

10 Zou W, Ni L, Lu Q, Zou C, Zhao M, Xu X, et al. Diabetes onset at 31-45 years of age is associated with an increased risk of diabetic retinopathy in type 2 diabetes. Sci Rep. 2016;6: 38113.

11 Khan R, Singh S, Surya J, Sharma T, Kulothunga V, Raman R. Age of onset of diabetes and its comparison with prevalence and risk factors for diabetic retinopathy in a rural population of india. Ophthalmic Res. 2019;61: $236-42$. 
12 Huo L, Magliano DJ, Rancière F, Harding JL, Nanayakkara N, Shaw JE, et al. Impact of age at diagnosis and duration of type 2 diabetes on mortality in australia 1997-2011. Diabetologia. 2018;61:1055-63.

13 Wang Y, Lin Z, Wen L, Rong SS, Ding XX, Li $\mathrm{D}$, et al. Design, methodology and baseline data of fushun diabetic retinopathy cohort study (fs-direct). Ophthal Epidemiol. 2020; 27:73-82.

14 American Diabetes Association. Diagnosis and classification of diabetes mellitus. Diabetes care. 2005;28(Suppl 1):S37-42.

15 Grading diabetic retinopathy from stereoscopic color fundus photographs--an extension of the modified airlie house classification. Etdrs report number 10. Early treatment diabetic retinopathy study research group. Ophthalmology 1991;98:786-806.

16 Wang FH, Liang YB, Zhang F, Wang JJ, Wei WB, Tao QS, et al. Prevalence of diabetic retinopathy in rural china: the handan eye study. Ophthalmology. 2009;116:461-7.

17 Xu J, Wei WB, Yuan MX, Yuan SY, Wan G, Zheng YY, et al. Prevalence and risk factors for diabetic retinopathy: the Beijing communities diabetes study 6. Retina. 2012;32:322-9.
18 Li YY, Yang XF, Gu H, Liu XP, Snellingen T, Liu NP. The beijing desheng diabetic eye study: rationale, design, methodology and baseline data. Int J Ophthalmol. 2018;11:10816.

19 Zhang J, Ma J, Zhou N, Zhang B, An J. Insulin use and risk of diabetic macular edema in diabetes mellitus: a systemic review and metaanalysis of observational studies. Med Sci Monit. 2015;21:929-36.

20 Raman R, Rani PK, Kulothungan V, Rachepalle SR, Kumaramanickavel G, Sharma T. Influence of serum lipids on clinically significant versus nonclinically significant macular edema: sn-dreams report number 13. Ophthalmology. 2010;117:766-72.

21 Klein BE, Myers CE, Howard KP, Klein R. Serum lipids and proliferative diabetic retinopathy and macular edema in persons with longterm type 1 diabetes mellitus: the wisconsin epidemiologic study of diabetic retinopathy. JAMA Ophthalmol. 2015;133(5):503-10.

22 Jew OM, Peyman M, Chen TC, Visvaraja S. Risk factors for clinically significant macular edema in a multi-ethnics population with type 2 diabetes. Int J Ophthalmol. 2012;5: 499-504.
23 Chiarelli F, Spagnoli A, Basciani F, Tumini S, Mezzetti A, Cipollone F, et al. Vascular endothelial growth factor (vegf) in children, adolescents and young adults with type 1 diabetes mellitus: relation to glycaemic control and microvascular complications. Diabet Med. 2000;17:650-6.

24 Zhong Q, Kowluru RA. Epigenetic modification of sod2 in the development of diabetic retinopathy and in the metabolic memory: role of histone methylation. Invest Ophthalmol Vis Sci. 2013;54:244-50.

25 Kowluru RA. Mitochondria damage in the pathogenesis of diabetic retinopathy and in the metabolic memory associated with its continued progression. Curr Med Chem. 2013;20:3226-33.

26 Reddy MA, Zhang E, Natarajan R. Epigenetic mechanisms in diabetic complications and metabolic memory. Diabetologia. 2015;58: 443-55.

27 Fenwick E, Rees G, Pesudovs K, Dirani M, Kawasaki R, Wong TY, et al. Social and emotional impact of diabetic retinopathy: a review. Clin Exp Ophthalmol. 2012;40:27-38.

28 Kalantari S, Jafarinezhad A, Zohrevand B. Association of depression with type 2 diabetes and relevant factors. Adv Biomed Res. 2014;3: 244 